\title{
Typologie et fonctions morphosyntaxiques des préfixes verbaux ha- et -ha- en kirundi ${ }^{1}$ (JD62)
}

\section{Typology and morphosyntactic functions of the verbal prefixes ha- and -ha- in Kirundi (JD62)}

\author{
Tipologia e funções morfossintáticas dos prefixos verbais ha- e - \\ ha- em kirundi (JD62)
}

\author{
Ernest Nshemezimana* \\ ernesto7620@gmail.com \\ Université du Burundi \\ Ferdinand Mberamihigo **
}

\begin{abstract}
${ }^{1}$ Dans la classification référentielle des langues bantoues actualisée par MAHO (2009), le kirundi est répertorié sous le sigle JD62. II est utilisé par environ 8.053.574 habitants du Burundi, selon les dernières informations fournies lors du recensement de la population effectué en 2008. II s'agit d'une langue en intercompréhension avec certaines langues de son voisinage immédiat, dont principalement, le kinyarwanda (JD61) parlé au Rwanda, ainsi que quelques langues en usage sur le territoire tanzanien, dans les régions frontalières du Burundi (sud et nord-est), comme le kiha (JD66) parlé dans le Buha (sud), le kihangaza (JD65) et le kishubi (JD64) dans le Bushubi (nord-est). Le kirundi se rapproche aussi au kivinza (JD67) parlé à Uvinza en Tanzanie au sud du Buha.

* Ernest Nshemezimana travaille actuellement comme professeur à l'Université du Burundi, située à Bujumbura, la capitale du Burundi. II y enseigne les cours de linguistique du Kirundi et du Kiswahili. II a obtenu son diplôme de Master à l'Université Catholique de Louvain en 2010, et son diplôme de Doctorat en Langues et Cultures Africaines à l'Université de Gand, en 2016. Son domaine de recherche couvre la description et l'analyse des langues bantoues, avec un intérêt particulier pour le kirundi (JD 62), la morphosyntaxe et la structure informationnelle.

Ernest Nshemezimana works currently as a Lecturer teaching linguistics courses applied to Kirundi and Kiswahili at the University of Burundi located in Bujumbura, capital city of Burundi.

He obtained his master's degree in linguistics from the Catholic University of Louvain in 2010, and his $\mathrm{PhD}$ in African Languages and Cultures at the University of Ghent in 2016. His research interests lie in the description and analysis of Bantu languages, with a special focus on Kirundi (JD62), morphosyntax and information structure.

Ernest Nshemezimana atualmente trabalha como professor, ministrando cursos de linguística aplicados ao kirundi e ao kiswahili na Universidade do Burundi, em Bujumbura, capital do país. Obteve o grau de mestre em Linguística na Universidade Católica de Louvain, em 2010, e o grau de doutor em Línguas e Culturas Africanas na Universidade de Ghent, em 2016. Seus interesses de pesquisa concentram-se na descrição e na análise das línguas bantu, com ênfase especial no kirundi (JD62), na morfossintaxe e em estruturas da informação.

** Ferdinand MBERAMIHIGO est professeur à l'Université du Burundi. II a obtenu son diplôme de Doctorat en Langues et Cultures Africaines à l'Université de Gand, en Belgique, en 2014.

Son domaine de recherche comprend la linguistique africaine, avec un intérêt particulier pour le kirundi (la langue nationale du Burundi), la linguistique de corpus, la lexicologie et la lexicographie, ainsi que la sémantique.

Ferdinand Mberamihigo is a lecturer at the University of Burundi. He obtained his PhD in African Languages and Cultures at Ghent University in Belgium in 2014. His areas of interest comprise African Linguistics, with a special focus on Kirundi (the national language of Burundi), corpus linguistics, lexicology and lexicography, and semantics.

Ferdinand Mberamihigo é professor na Universidade do Burundi. Obteve o grau de doutor em Línguas e Culturas Africanas na Universidade de Ghent, Bélgica, em 2014. Suas áreas de interesse incluem a Linguística Africana, com enfoque especial no kirundi (língua nacional do Burundi), na linguística de corpus, na lexicologia/lexicografia e na semântica.
\end{abstract}


fmberamihigo@gmail.com

Université du Burundi

RESUME: Cet article étudie la typologie et le fonctionnement morphosyntaxique des préfixes verbaux ha-/-ha- attestés en kirundi. Empruntant une démarche du corpus, nos résultats d'analyse font état de deux types de ha: un préfixe locatif référant à la classe locative 16 et un préfixe non-référentiel à caractère explétif. Ces derniers ont des usages et une fréquence d'emploi assez différents. Le plus dominant dans les emplois est le préfixe ha explétif, surtout lorsqu'il est sujet du verbe. Celui-ci est essentiellement utilisé comme marqueur du sujet sur le verbe dans certaines structures à sujet inversé, après que ce dernier ait perdu la capacité d'assurer l'accord du verbe suite à sa délocalisation de sa position canonique. Parfois, ce préfixe verbal se présente comme l'objet pronominal explétif en conférant une valeur existentielle au verbe. Pour le préfixe locatif, ses fonctions varient entre marqueur d'accord verbal et marqueur pronominal des arguments sur le verbe donné.

MOTS CLES: Kirundi. Fonctions morphosyntaxiques. Préfixes locatifs. Suffixes locatifs.

ABSTRACT: This article deals with the typology and the morphosyntactic properties of the verbal prefixes ha-/-ha- attested in Kirundi. Based on a corpus, it reveals two types of ha: a locative prefix referring to locative class 16 and a non-referential expletive prefix. These markers have different uses and are different in frequency.

The most frequently used is the expletive prefix ha, especially when it works as the subject concord of the verb. It is mainly used as a subject concord of the verb in some structures with inverted subject, after the latter has lost the capacity to lead the agreement of the verb, given that it has been delocated from its canonic position. Sometimes it stands as an expletive pronominal object, imparting an existential reading to the verb. As for the locative prefix, it functions either a verbal agreement marker or as a pronominal marker of the arguments on the verb.

KEYWORDS: Kirundi. Morphosyntactic functions. Locative prefixes. Locative suffixes.

RESUMO: Este artigo apresenta um estudo da tipologia e das propriedades morfossintáticas dos prefixos verbais ha- e -ha- atestados no kirundi. Baseados em um corpus, os resultados revelam dois tipos de ha: um prefixo locativo que se refere à classe locativa 16 e um prefixo não referencial de caráter expletivo. Esses marcadores apresentam usos diferentes bem como são diferentes em termos de frequência. $O$ de emprego mais frequente é o prefixo expletivo ha, em especial quando funciona como sujeito do verbo. É principalmente usado como marcador do sujeito em algumas estruturas com sujeito invertido, depois que este perde a capacidade de assegurar a concordância do verbo, dado o seu deslocamento de sua posição canônica. Às vezes, apresenta-se como um objeto pronominal expletivo, conferindo uma leitura de valor existencial ao verbo. Quanto ao prefixo locativo, sua função varia entre a de marcador da concordância verbal e a de marcador pronominal dos argumentos para o verbo.

PALAVRAS-CHAVE: Kirundi. Funções morfossintáticas. Prefixos locativos. Sufixos locativos. 


\section{Introduction}

Les points de vue dans la littérature sont divergents quant à la nature et la fonction de deux préfixes verbaux ha en kirundi : l'un se trouvant en position préverbale "ha-" et l'autre en position préradicale “-ha-". La majorité des travaux antérieurs leur reconnaissent l'unique statut d'éléments référentiels en les décrivant comme référant à la classe nominale locative 16 (BUKURU, 2003; MEEUSSEN, 1959; MISAGO, 2018; NTAHOKAJA, 1994; SABIMANA, 1986). Rares sont des travaux qui s'interrogent sur leur caractère explétif. Ceux qui en parlent, notamment NDAYIRAGIJE (1999), MORIMOTO (2000) ou VAN DER WAL (2009), s'arrêtent au seul ha- préverbal tandis qu'ils ne s'empêchent pas de le référer en même temps à la classe locative 16, même en emploi explétif. D'autres vont jusqu'à nier catégoriquement la valeur référentielle de ce dernier en le prenant plutôt comme pronom impersonnel (TUYUBAHE, 2017). Aucun des travaux sur le kirundi n'avait jusqu'ici proposé une étude détaillée de ces éléments morphologiques dans la langue.

Le présent travail consistera à étudier systématiquement ces morphèmes aux deux plans formel et fonctionnel. Sur le plan formel, nous nous interrogeons sur leurs différentes valeurs grammaticales en établissant une catégorisation typologique conséquente. Sur le plan fonctionnel, notre étude vise une description détaillée des différentes fonctions morphosyntaxiques qu'assurent ces morphèmes dans la langue. Cette étude exploite une démarche méthodologique reposant sur le corpus. Nous présentons ci-après la méthodologie qui sera utilisée.

\section{Méthodologie}

Cet article s'inscrit dans le cadre des études en linguistique de corpus. C'est une étude basée sur le corpus (corpus-based) au sens où l'entend Tognini-Bonelli (2001: 2). Nous exploitons les données d'un corpus de textes en kirundi, qui s'étendent sur environ dix décennies, trouvable dans le Centre d'Etudes bantoues de l'Université de Gand (BantUGent). Plusieurs études en linguistique ont été effectuées à partir d'une version du même corpus, à savoir Bostoen et al. (2012), Mberamihigo (2014), Mberamihigo et al. (2016), Nshemezimana (2016), Lafkioui et al. (2016), Nshemezimana \& Bostoen (2017), Devos et al. (2017), Misago (2018), Misago et al. (2019), Mberamihigo et al. (2020) et Nshemezimana (2021). Il s'agit d'un corpus dont la compilation a été réalisée à l'université de Gand sous la supervision des professeurs 
Koen Bostoen et Gilles-Maurice de Schryver lors des travaux de recherche doctorale de Mberamihigo (2014), Nshemezimana (2016) et MISAGO (2018). Ce corpus regroupe actuellement des productions langagières totalisant 3.314.339 tokens dont 2.525 .512 tokens (soit $76 \%$ ) relèvent du langage écrit et 788.827 tokens (soit $24 \%$ ) du langage oral. Les détails sur son état actuel sont présentés dans MISAGO (2018:3744).

La compilation des données sur lesquelles porte cette étude a été réalisée en trois étapes, par l'auteur.

La première étape a consisté à extraire, à l'aide du logiciel WordSmith Tools 7 (https://www.lexically.net/wordsmith/), un échantillon aléatoire et représentatif de phrases attestant les préfixes en question, à la fois dans les parties du corpus issues du langage écrit et oral. De même, en vue de travailler sur des échantillons équilibrés et faciles à gérer, nous avons essayé de réduire la taille de chaque partie de l'échantillon en jouant sur le nombre d'occurrences de ha- et de -ha- obtenues pour chaque partie du corpus, et cela, compte tenu aussi de la taille de chacune d'elles. Cette démarche a ainsi consisté à sélectionner aléatoirement une phrase toutes les vingt phrases dans les données écrites contre une phrase toutes les sept phrases dans les données orales. L'opération a abouti à 4198 attestations pour le sous-corpus écrit et 4154 pour le sous-corpus oral.

A la seconde étape, nous avons fait le dépouillement du premier échantillon pour en exclure les occurrences de "ha" ne représentant ni l'un ni l'autre des deux préfixes verbaux, comme par exemple dans aba-ha-bara "concubines", aba-ha-nuzi "conseillers", etc. A l'issue de ce dépouillement, nous nous sommes retrouvés avec 1107 attestations pour le sous-corpus écrit et 1292 attestations pour le sous-corpus oral où ha correspond à un préfixe verbal.

A la troisième étape, nous avons procédé à l'extrapolation des résultats obtenus à partir de l'échantillon à l'ensemble du corpus. Sur 2.525 .512 tokens, le sous-corpus écrit contient 22140 occurrences des préfixes verbaux ha-/-ha- (c'est-à-dire $1107 \mathrm{X}$ 20), tandis que le sous-corpus oral en contient 9044 (c'est-à-dire $1292 \times 7$ ) sur 788.827 tokens. Exprimé en nombre d'occurrences par un million de tokens, nous pouvons constater que les mots contenant les préfixes verbaux ha-/-ha- sont plus fréquents à l'oral qu'à l'écrit : 11465 occurrences par million de mots dans le langage oral contre 8766 dans le langage écrit. Ci-dessous, nous présentons la distribution typologique de 
ces préfixes verbaux tels que trouvés dans le corpus, avant d'entamer la description de leurs fonctions morphosyntaxiques.

\section{Distribution typologique des préfixes verbaux ha-/-ha-}

Les préfixes verbaux ha sont des morphèmes qui, dans le verbe, apparaissent avant le radical. Ils sont donc à classer parmi les affixes verbaux préfixés au radical du verbe. L'analyse des données retenues pour les deux sous-corpus met en exergue deux catégories de préfixes verbaux "ha" en kirundi. La première catégorie est celle des préfixes verbaux référentiels qui réfèrent à la classe nominale locative 16 (soit PV_LOC). Ce sont des éléments qui co-réfèrent à un circonstant locatif dénotant une localisation spatiale comme dans les verbes harazwi "est connu" en (1) et baraharimye "on y a cultivé" ou barahatéeye "ils y ont cultivé" en (2), où les morphèmes ha renvoient aux circonstants locatifs dénotant le lieu, à savoir respectivement aho hantu "ce lieu" (1) et ahari amasato "là où il y a des pythons" (2). Ils peuvent aussi référer à une localisation temporelle comme dans le verbe haciye "venaient de passer" en (3), où ha- renvoie à l'idée du temps correspondant aux années qui venaient de passer.

(1) Aho hantu harazwi ko ari ku gasaka ka Inarunyonga.

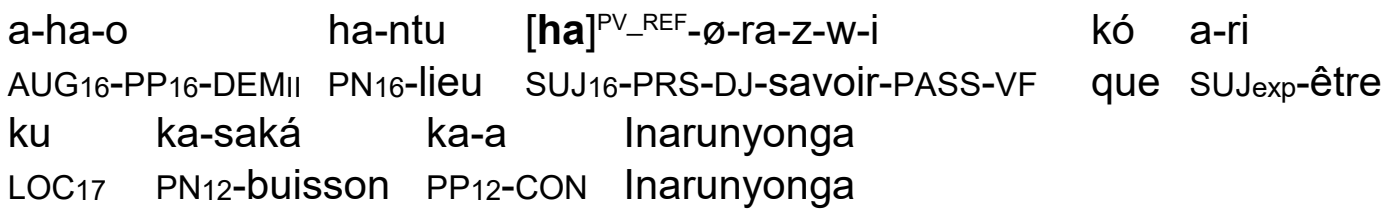

"Ce lieu est connu que c'est au buisson de Inarunyonga."

(IragiNdanga, culture traditionnelle, 2000s)

(2) Ahari amasato ubu baraharimye, barahateye ibitoke.

a-ha-á-ri a-ma-sáto ubu ba-á-ra-[ha] ${ }^{\mathrm{PV}}{ }^{\mathrm{REF}}$-rim-ye

AUG16-PP16-PE-être AUG6-PN6-python maintenant SUJ2-PE-DJ-OBJ16-cultiver-PRF ba-á-ra-[ha] ${ }^{\mathrm{PV}}{ }^{\mathrm{REF}}$-téer-ye i-bi-tooke

SUJ2-PE-OBJ16-planter-PRF AUG8-PN8-bananier

"Là où il y avait des pythons on y a maintenant cultivé, on y a planté des bananiers."

(Nibaruta_Ubuhinga, Culture traditionnelle, 2010s)

(3) Aho mboneye haciye imyaka itari mike, narahavuye ndaza gutabara.

$\begin{array}{llcl}\text { a-ha-ó } & \text { N-bón-ir-ye } & \text { [ha] }^{\text {PV_REF-ci-ye }} & \text { i-mi-aka } \\ \text { AUG-PP16-PRCS } & \text { SUJ1sG-voir-APPL-PRF.REL } & \text { SUJ16-passer-PRF } & \text { AUG4-PN4-année } \\ \text { i-ta-ri } & \text { mi-ke N-á-ra-havu-ye } & \text { N-ra-əz-a } & \end{array}$




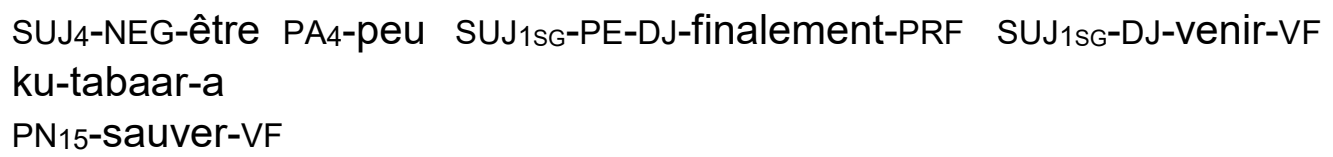

"Quand j'ai vu que plusieurs années venaient de passer, je suis finalement venu intervenir."

(Ubwuzure.Bushasha, Religion, 1960s)

La seconde catégorie referme des préfixes verbaux non référentiels qui fonctionnent comme des éléments explétifs (soit PV_EXP). Ceux-ci sont illustrés dans les exemples (4) et (5) ci-après.

(4) lyo havutse amahasa vyari ibindi kuko abarundi baratinya amahasa.

$\begin{array}{llll}\text { lyó } & \text { [ha] }^{\text {Pv_EXP-Vúuk-ye }}{ }^{H} & \text { a-ma-hása } & \text { bi-á-ri } \\ \text { Quand SUJEXP-naitre-PRF.REL } & \text { AUG6-PN6-jumeau } & \text { SUJEXP-PE-être } \\ \text { i-bi-ndi } & \text { kukó } & \text { a-ba-rundi } & \text { ba-ø-ra-tíiny-a } \\ \text { AUG8-PP8-autre (chose) } & \text { puisque } & \text { AUG2-PN2-burundais } & \text { SUJ2-PRS-DJ-craindre-VF } \\ \text { a-ma-hása } & & & \\ \text { AUG6-PN6-jumeau } & & & \end{array}$

"Lorsqu'il était né des jumeaux, c'était autre chose puisque les Burundais craignent les jumeaux."

(Nduwayo_Ubushobozi, Culture traditionnelle, 2010s)

(5) (...) kiretse ko n'ubusho buhasanzwe bushaje.

$\begin{array}{lllll}\text { kiretse kó } & \text { n' } & \text { u-bu-sho } & \text { bu-[ha] }{ }^{\text {PV_EXP-sang-u-ye }}{ }^{H} \\ \text { sauf que } & \text { même } & \text { AUG14-PN14-cachot } & \text { SUJ14-OBJEXP-trouver-PASS-PRF.REL } \\ \text { bu-sáaz-ye } & & & \end{array}$

SUJ14-veillir-PRF

"(...) sauf que même les cachots qui existent déjà sont vieux."

(ljwi rya CNIDH 1, Paix, 2010s)

Les exemples précédents montrent que dans chaque catégorie ces préfixes verbaux occupent deux positions alternatives. Les uns sont identifiés en position initiale du verbe, comme dans harazwi (1), haciye (3) et havutse (4) et les autres au milieu, c'est-à-dire en position préradicale, comme dans baraharimye/barahatéeye (2) et buhasanzwe (5). Pour mieux les distinguer ici, nous proposons de désigner les premiers par "préfixe préverbal" (soit P_PréVRB) et les seconds par "préfixe préradical' (soit P_PréRAD).

L'analyse des données du corpus montre que, de manière générale, les préfixes ha explétifs dominent dans les emplois en kirundi. Cette dominance est encore plus importante pour le ha- explétif de type P_PréVRB. Les attestations de ce dernier s'élèvent à $65 \%$ à l'oral et $60 \%$ à l'écrit. 
Les deux types de préfixes verbaux ha-/-ha- étant identifiés, il revient maintenant à décrire leurs fonctions morphosyntaxiques dans cette langue bantoue.

\section{Fonctions morphosyntaxiques des préfixes verbaux ha-/-ha-}

Les préfixes verbaux ha-/-ha- répondent à différentes fonctions morphosyntaxiques dans le verbe en kirundi. Les données du corpus nous ont permis d'en identifier trois, à savoir (i) assurer l'accord entre le verbe et son sujet grammatical, (ii) représenter anaphoriquement, en tant que sujet pronominal (SUJ_PRO) ou objet pronominal (OBJ_PRO), le sujet ou l'objet lexical disloqué ou absent dans la structure de surface ou (iii) jouer le rôle de sujet explétif (SUJ_EXP) ou d'objet explétif (OBJ_EXP). La figure ci-dessous visualise la distribution statistique des types de préfixes ha fonctionnels en kirundi et différentes fonctions occupées par ces derniers dans cette langue bantoue.

Figure 1: Distribution statistique des types de préfixes ha et leurs fonctions morphosyntaxiques en kirundi

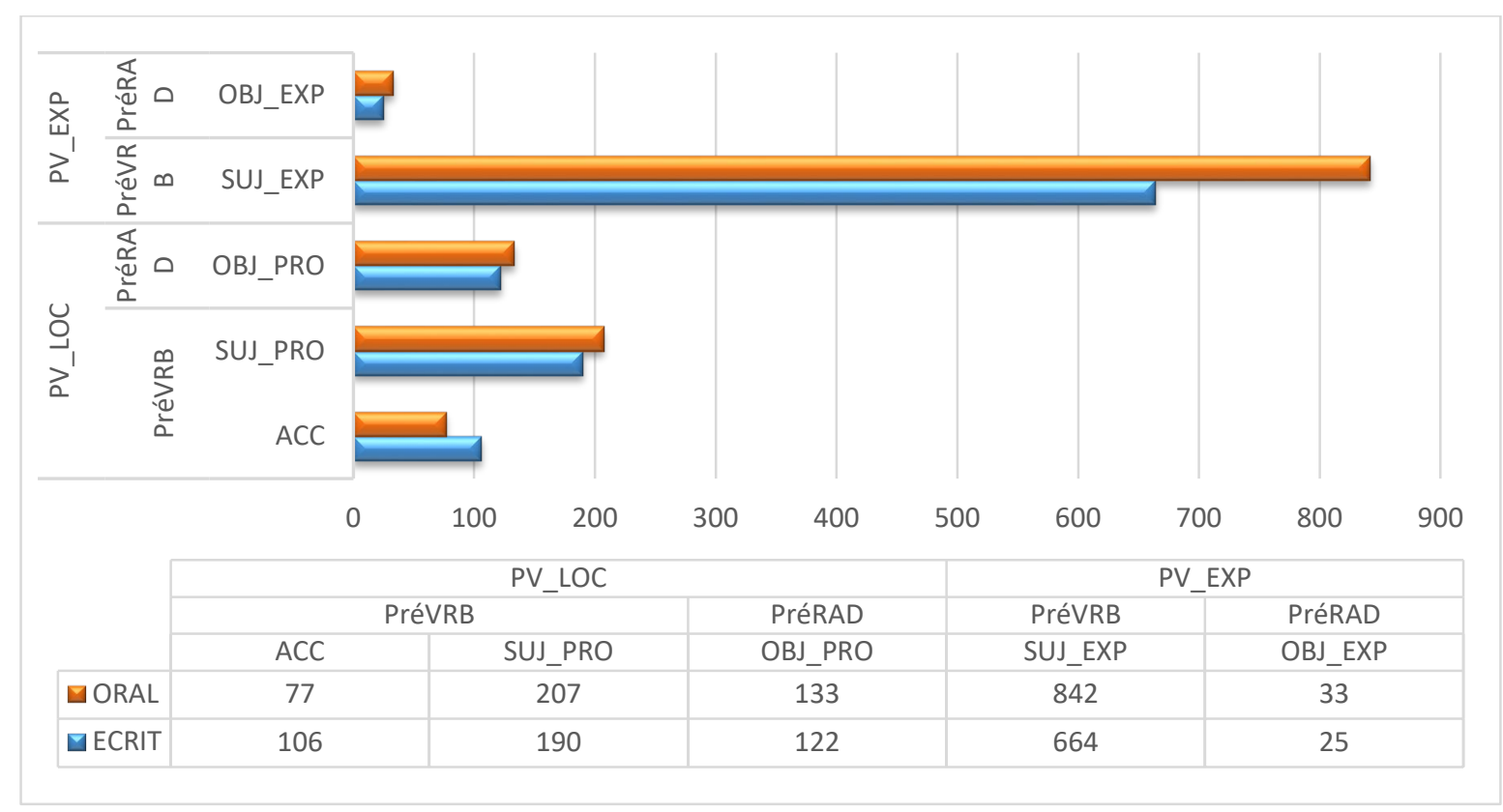

Dans les sections qui suivent, nous nous proposons de décrire systématiquement comment ces préfixes verbaux fonctionnent sur le plan morphosyntaxique, tel qu'il a été signalé dans les objectifs du présent article. 


\subsection{Fonctions des préfixes verbaux locatifs (PV_LOC)}

Les préfixes verbaux locatifs ont en tant qu'éléments référentiels des fonctions qui varient selon le constituant syntaxique auquel ils réfèrent. Ils fonctionnent tantôt comme marqueur (d'argument) pronominal du sujet (SUJ_PRO) ou d'objet (OBJ_PRO), lorsque ces derniers sont disloqués ou implicites, tantôt comme marqueur d'accord (ACC) entre le verbe et son sujet grammatical (locatif) avec lequel il forme une même relation syntaxique.

Dans certaines langues bantoues, la distinction entre le marqueur d'accord verbal et le marqueur d'argument est parfois difficile à distinguer. Par exemple en chichewa (N30), BRESNAN et MCHOMBO (1987) notent que le marqueur du sujet fonctionne parfois à la fois comme la marque d'accord et le sujet pronominal anaphorique au sujet lexical. Cet état de choses est aussi rapporté en kiswahili vis-àvis du marqueur d'objet. Pour certains auteurs, celui-ci s'emploie toujours comme pronom (GIVON, 1976; HYMAN, DURANTI, 1982; WALD, 1997) tandis qu'il est décrit ailleurs comme la marque d'accord entre le verbe et son objet lexical (VITALE, 1981).

En kirundi, les données du corpus font état de deux préfixes verbaux locatifs différents, un préverbal (ha-) et un autre préradical (-ha-) qui marquent respectivement le sujet et l'objet dans le verbe. Nous les présentons ci-après.

\section{a) Ha-, marqueur du sujet locatif}

Le préfixe locatif marqueur du sujet a une double fonction en kirundi. Il est d'abord le seul à pouvoir jouer le rôle d'accord (ACC) verbal, en liant syntaxiquement le verbe et son sujet. Le cas échéant, il fait régulièrement partie de la flexion verbale et le constituant syntaxique auquel il se rapporte, c'est-à-dire le sujet du verbe, est obligatoirement interne à la relation syntaxique qu'il forme avec le reste de l'énoncé. Mais lorsque ce dernier n'apparait pas dans cette relation syntaxique, c'est-à-dire s'il est disloqué ou absent dans la structure de surface, le marqueur du sujet a le statut de pronom anaphorique au sujet lexical en question. Précisons que la reprise anaphorique du sujet externe par un pronom co-référent dans le verbe est obligatoire en kirundi. II en est de même pour l'objet lexical externe (NSHEMEZIMANA, 2016).

Marqueur d'accord verbal, le préfixe ha- co-réfère régulièrement avec le sujet locatif interne à la relation syntaxique en cours, comme dans (6) ci-après :

\section{(6) Ndazi ko aha hantu hashushe kuri uno mugoroba.}




$\begin{array}{lllll}\text { N-ra-zi } & \text { kó } & \text { a-ha-a } & \text { ha-ntu } & \text { [ha] }^{\text {Acc }} \text {-shuh-ye } \\ \text { SUJ1sG-DJ-savoir } & \text { que } & \text { AUG16-PP16-DEM। } & \text { PN16-lieu } & \text { SUJ16-être.chaud-PRF } \\ \text { kuri } & \text { U-nó } & \text { mu-goroba } & & \\ \text { PLOC17 }_{17} \text { PP3-DEM }_{1 /} & \text { PN3-Soirée } & & \end{array}$

"Je sais que ce lieu est chaud cette soirée."

(IcaGatandatu, Religion, 2010s)

Dans l'exemple précédent, le verbe hashushe "est chaud" et son sujet locatif aha hantu "cet endroit-ci" forment une même relation syntaxique et le préfixe verbal ha- constitue l'élément d'accord entre les deux constituants syntaxiques. En kirundi, ceci a lieu dans les constructions de base, c'est-à-dire construisant le verbe et ses arguments dans leurs positions par défaut, comme en (6), ou dans celles à sujet postverbal, appelées souvent "inversion locative" (MARTEN, VAN DER WAL, 2014; ZERBIAN, 2006), comme celle en (7) ci-après :

(7) Ku murwa mukuru w'intara ya Makamba hahuriye abakenguzamateka n'abaserukiye abajenama b'amakomine (...).

[Ku mu-rwa mu-kuru u-a i-n-tara i-a

PLOC17 PN3-chef.lieu PN3-grand PP3-CON AUG9-PN9-province PP9-CON

Makamba] ${ }^{\mathrm{LOC}}{ }^{\mathrm{NT}} \quad[\mathrm{ha}]^{\mathrm{ACC}}$-a-a-hur-ir-ye a-ba-kenguzamateka

Makamba sUJ16-PR-DJ-rencontre-APPL-PRF AUG2-PN2-senateur

n' a-ba-serukir-ye a-ba-jenama ba-a

et AUG2-PP2-représenter-PRF AUG2-PN2-conseiller PP2-CON

a-ma-komine (...)

AUG6-PN6-commune

"Au chef-lieu de la province Makamba se sont rencontrés les sénateurs et les représentants des conseillers communaux."

(Senat0910-27, Politique, 2000s)

Dans (7), le sujet du verbe abakenguzamateka n'abaserukiye abajenama b'amakomine "les sénateurs et les représentants des conseillers communaux" a changé de place avec le complément locatif ku murwa mukuru w'intara ya Makamba "au chef-lieu de la province Makamba" de sorte que l'un occupe la position canonique de l'autre. Cette opération a donné lieu à la construction d'inversion locative évoquée ci-dessus. Ceci n'est cependant possible que si le complément locatif est régi par le verbe, c'est-à-dire s'il est compté parmi les arguments du verbe avec le statut d'objet locatif (MISAGO, 2018; NSHEMEZIMANA, 2016). Or, dans cet exemple, ce statut lui est rendu par la présence de l'applicatif -ir- dans le verbe, qui est identifié en langues 
bantoues parmi les dérivatifs augmentant souvent la valence du verbe (GUTHRIE, 1962). L'applicatif a établi ici un lien rectionnel entre le verbe et ce complément locatif désormais en préposition. Dans cette nouvelle position, ce complément locatif est devenu le sujet grammatical du verbe, en commandant l'accord du verbe hahuriye "s'y sont rencontrés" : le verbe prend son accord en classe locative 16 plutôt qu'en classe 2 de son sujet logique qui se trouve désormais en position postverbale.

Lorsqu'il réfère à un argument locatif implicite (8) ou externe (9) à la structure de surface, le préfixe locatif ha- a le statut de pronom locatif. Le corpus montre une nette prédominance d'attestations d'un tel préfixe ha se présentant sous forme d'un pronom par rapport à ses attestations lorsqu'il fonctionne comme élément d'accord, avec un taux de fréquence évalué à $74,64 \%$ à l'écrit et $81,53 \%$ à l'oral.

(8) (...) dushitse, dusanga hakoraniye abatware batari bakeyi.

(...) tu-shik-ye tu-sang-a [ha] ${ }^{\text {RRO-kóran-ir-ye }}$

(....) SUJ1PL-arriver-PRF SUJ1PL-trouver-VF SUJ16-se.rassembler-APPL-PRF

a-ba-twaáre ba-ta-ri ${ }^{H}$ ba-kéeyí

$\mathrm{AUG}_{2}$-PN2-dirigeant SUJ2-NEG-être.REL PA2-peu

"Arrivés, nous y trouvons rassemblé un nombre non moins important de dirigeants."

(Ubuzima, Histoire, 1990s)

(9) Aho akuye ikirenge, wogira haciye umusakanyiga.

[a-ha-ó a-kúur-ye i-ki-renge] ${ }^{\text {LOC_EXT }}$ u-o-gir-a

AUG16-PP16-PRCS SUJ3sG-retirer-PRF AUG7-PN7-pied SUJEXP.-POT-dire-VF

$\left[\right.$ ha ${ }^{\text {PRO-}} \varnothing$-ci-ye u-mu-sakanyiga

SUJ16-PRS-passer-PRF AUG3-PN3-putois

Où il passe, on dirait que c'est un putois qui vient d'y passer.

(Igitabu c'umwaka wa2, Education, 1990s)

Le préfixe locatif ha- au statut de pronom peut être associé avec un suffixe locatif post-final. En kirundi, le suffixe locatif post-final est un des quatre pronoms -hó, -mwó, -kó et -yó, qui co-réfèrent respectivement aux prépréfixes locatifs ha (cl.16), mu (cl.17), ku (cl.18) et $\boldsymbol{i}$ (cl.19) (MEEUSSEN, 1959: 64). Ces derniers sont morphologiquement composés du préfixe locatif co-référent et du thème ó, comme mwó /mu-ó/ dans harabónekamwo "s'y trouve" dans l'exemple (10) ci-dessous.

(10) Muri aya mavuriro, harabonekamwo umwahwa.

murí a-a-a ma-vuuriro[ha] ${ }^{\text {PRO-ra-bónek-a-mwó }}$

PLOC18 AUG6-PP6-DEMI PN6-centre.de.santé SUJ16-DJ-être observé-VF-PSTF18 u-mu-aáhwa

AUG3-PN3-médicament.traditionnel 
"Dans ces centres de santé-ci, s'y trouve un médicament traditionnel." (Akanovera, Education, 2000s)

Sur le plan fonctionnel, le suffixe locatif consiste à renforcer le caractère pronominal du préfixe ha-/-ha- dans la mesure où, combiné avec ce dernier dans le même verbe, il exprime lui-même un renvoi anaphorique au circonstant locatif extrait de la structure de la phrase. Mais à la différence du pronom locatif ha- qui réfère régulièrement à la classe 16 , le suffixe locatif réfère à la classe du prépréfixe locatif introduisant le circonstant locatif donné. A titre d'exemple, -mwo en (10) renvoie au prépréfixe locatif muri (cl.18) à l'initiale du circonstant locatif muri aya mavuriro "dans ces centres de santé". La présence du suffixe locatif dans le verbe est un indice absolu qui témoigne que le sujet locatif correspondant est externe à la relation syntaxique de la phrase. Par ailleurs, ceci est aussi attesté ailleurs dans le domaine linguistique bantou, comme par exemple en tsootso, un des dialectes du luyia (JE32) parlé au Kenya, où le suffixe locatif est rapporté comme indiquant régulièrement l'absence du syntagme nominal locatif correspondant dans la relation syntaxique en présence (DALGISH, 1976: 141).

En kirundi, l'usage du suffixe locatif a une particularité sur le plan syntaxique : il rend au sujet locatif, alors externe, une liberté syntaxique, c'est-à-dire lui permettant de se placer en début (11a), au milieu (11b) ou en fin (11c) de phrase.

\section{(11) Murí aka gakino, harimwó ibihiímba bitatu.}

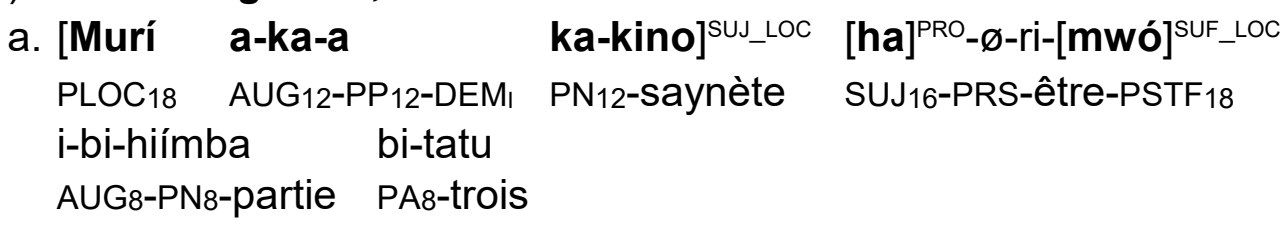

"Dans cette saynète, s'y trouvent trois parties." (Amazi, Théâtre, 1970s)

b. [ha] ${ }^{\mathrm{PRO}}-\varnothing-$-ri-[mwó] $]^{\mathrm{SUF}} \_$LOC

[murí a-ka-a ka-kino] $]^{\mathrm{SUJ} \_ \text {Loc }}$

SUJ16-PRS-être-PSTF18 PLOC18 AUG12-PP12-DEM! PN12-Saynète

i-bi-hímba bi-tatu

AUG8-PN8-partie PA8-trois

"S'y trouvent, dans cette saynète, trois parties." (adapté à partir de (11a))

c. [ha] $]^{\text {PRO-}}$-ø-ri-[mwó] ${ }^{\text {SUF_Loc }}$ i-bi-hiímba

bi-tatu [murí a-ka-a

SUJ16-PRS-être-PSTF18 AUG8-PN8-partie PA8-trois PLOC18 AUG12-PP12-DEM ka-kino] ${ }^{\text {sUJ_LOC }}$ 
PN12-saynète

"S'y trouvent trois parties, dans cette saynète."

(adapté à partir de (11a))

Autrement, le sujet locatif externe ne peut pas précéder directement le verbe. II est soit séparé de celui-ci par un autre constituant syntaxique comme en (9) ou bien placé en position postverbale; sinon il reçoit une lecture différente. Cette remarque est d'autant plus importante dans la mesure où le suffixe locatif est, compte tenu de ce qui précède, le seul critère qui permet d'identifier le sujet locatif en début d'énoncé comme étant interne (si absent) ou externe (si présent).

L'emploi simultané du préfixe locatif et du suffixe locatif dans un verbe est souvent motivé par le fait que ce dernier apporte une précision que le préfixe ha- ne pourrait pas exprimer concernant le lieu d'affectation du procès désigné par le verbe, c'est-à-dire en indiquant si celui-ci est localisé parmi (avec -mwo) ou sur (avec -ko) le référent locatif donné ou à l'intérieur (-yo, -mwo) de celui-ci ou alors une référence existentielle (-ho). De tous les emplois des suffixes locatifs attestés en kirundi, le corpus présente une nette dominance de -mwo, avec 95,74\% (à l'écrit) et $66,15 \%$ (à l'oral) des attestations globales. Cela est lié selon nous au fait que celui-ci couvre des emplois variés de façon qu'il peut dans certains contextes alterner avec le suffixe -yo (cl.19), tel qu'illustré en (12a-b) ci-après:

(12) Aha harimwo iki?

\begin{tabular}{|c|c|}
\hline a-ha-a & ha-ri-mwó \\
\hline $\begin{array}{l}\text { AUG16-PP16-DEMı } \\
\text { a-ha-a }\end{array}$ & $\begin{array}{l}\text { SUJ16-être-PSTF18 } \\
\text { ha-ri-yó }\end{array}$ \\
\hline AUG16-PP16-DEM। & SUJ16-être-PSTF 19 \\
\hline
\end{tabular}

(RPA_JP_2014i, Informations, 2010s)

Dans d'autres emplois, le suffixe locatif intervient comme élément de désambiguïsation permettant de distinguer le préfixe locatif ha- de celui non référentiel, comme nous le décrivons au point 4.2 du présent article.

\section{b) Ha, marqueur d'objet locatif}

Lorsque dans le verbe le préfixe locatif ha apparait en position préradicale, il a le statut grammatical du marqueur pronominal de l'objet locatif du verbe disloqué (13) ou 
non exprimé (14) dans la structure de surface. Le corpus montre que tel type d'élément est attesté avec une faible fréquence dans la langue comparativement au marqueur pronominal du sujet locatif. Les emplois de ce dernier dépassent significativement ceux de l'objet locatif pronominal, avec un taux de fréquence évalué à plus de $60 \%$ à l'écrit comme l'oral.

(13) (...) ahasanga, mw'iyo Seminari y'i Mugera, Padri D'HERVE akiri Padri mukuru.

$\begin{array}{llll}\text { a-[ha] }{ }^{\text {PRO-sang-a }} & \text { [mw' }^{\prime} & \text { i-i-o } & \text { Seminari } \\ \text { SUJ3sG-OBJ16-trouver-VF } & \text { LOC } 18_{18} & \text { AUG9-PP9-DEM II } & \text { séminaire }\end{array}$

\begin{tabular}{|c|c|c|c|c|c|}
\hline i-a & $\mathbf{i}$ & Mugera] ${ }^{\text {OBJ_LOC }}$ & Padri & D'HERVE & a-ki-ri \\
\hline PP9-CON & $\mathrm{LOC}_{19}$ & Mugera & prêtre & D’herve & SUJ3sG-PERS-être \\
\hline
\end{tabular}

"Il y trouva, dans ce même séminaire de Mugera, Prête D’Herve alors curé." (Umwepiskopi, Histoire, 1960s)

(14) Aho zoba zitahasinzikaje, zahashizemwo umugera.
a-ha-ó
zi-o-ba-a
zi-ta-ha-sinzikaz-ye
AUG16-PP16-PRCS SUJ10-POT-être-VF SUJ10-NEG-OBJ16-perdurer-PRF
zi-á-[ha] ${ }^{\text {PRO }}$-shir-ye-mwó u-mu-gera.
SUJ10-PE-OBJ16-mettre-PSTF18 AUG3-PN3-virus
"Là où ils (problèmes) n'ont pas perduré y ont laissé un impact." (Mushingantahe, Paix, 2000s)

A la différence du marqueur du sujet dont le statut grammatical varie entre accord et pronom, le marqueur d'objet en kirundi est toujours un pronom. Autrement dit, l'objet n'assure généralement pas la concordance du verbe dans cette langue bantoue, ce qui vaut aussi pour l'objet locatif. De même, l'objet pronominal ne semble pas pouvoir être associé avec le suffixe locatif, comme le sujet pronominal décrit ci-haut. L'analyse des deux échantillons (oral et écrit) extraits du corpus n'atteste aucune co-occurrence des deux éléments morphologiques dans le même verbe. Toutefois, le corpus fait état des structures verbales où le suffixe locatif et le verbe qui le comporte constituent une forme verbale lexicalisée de sorte que leur séparation donne au verbe un autre sens lexical. Ainsi, avons-nous constaté que de telles formes verbales peuvent abriter, comme tant d'autres, le pronom locatif objet, comme dans le verbe barahasubiramwo 
en (15a). Ici, la suppression du suffixe locatif $-\boldsymbol{m} w \boldsymbol{w o}$ rendrait le verbe inapproprié au contexte comme illustré par le contre-exemple en (15b).

(15) (...) bagiye barahasubiramwo bagabura intara.

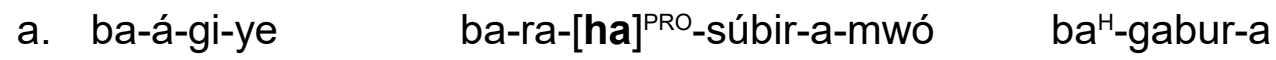

SUJ2-PE-aller-PRF SUJ2-DJ-OBJ16-répéter-VF-PSTF18 SUJ2.CJC-subdiviser-VF

i-n-táará

AUG10-PN10-province

"(...) ils y ont répétitivement réaménagé en subdivisant les provinces."

(Conversations_2015-01-05, Société, 2010s)

b. ba-á-gi-ye [ba-ra-ha-súbir-a-ø] (...).

SUJ2-PE-aller-PRF SUJ2-DJ-OBJ16-retourner-VF ( ...).

"lls y sont répétitivement retournés ...".

La raison d'être du non emploi simultané du préfixe locatif objet et du suffixe locatif dans le verbe réside dans le fait que les deux éléments peuvent alterner dans leur utilisation, comme illustré dans (16) ci-après.

(16) (...) asangayo abandi basore.
a. (...) a-sang-a-yo
a-ba-ndi
ba-sore
(...) SUJ1-trouver-VF-PSTF19
$A \cup G_{2}-P N_{2}$-autre
PN2-jeune.homme
"(...) il y trouva d'autres jeunes hommes."
(Es'iyo, Nouvelles, 1980s)
b. (...) a-[ha] $]^{\text {PRO }}$-sang-a
a-ba-ndi
ba-sore
(...) SUJ1-OBJ16-trouver-VF
AUG2-PN2-autre
PN2-jeune.homme
c. (...) a-sang-a-[mwó] ${ }^{\text {Loc18 }}(\ldots)$
d. (...) a-sang-a-[hó] ${ }^{\operatorname{Loc} 16}(\ldots)$
e. (...) a-sang-a-[kó] $]^{\operatorname{Loc} 17}(\ldots)$

Les exemples en (16a-e) montrent que l'objet pronominal locatif peut être utilisé à la place du suffixe locatif co-référent et vice versa pour exprimer une même entité ; ce qui implique que leur présence simultanée dans un même verbe constituerait une pure redondance.

\subsection{Les préfixes verbaux ha-/-ha- explétifs}

En usage explétif, les préfixes verbaux ha sont des éléments dépourvus de référentialité, qui ne renvoie à aucun référent locatif. Dans plus de $96 \%$ de ses 
attestations dans les deux sous corpus, il a la fonction du sujet explétif. Le reste, respectivement $3,63 \%$ à l'écrit et $3,77 \%$ à l'oral, sont des occurrences où il est objet explétif du verbe.

\section{a) Ha, marqueur du sujet explétif}

Plus haut, nous avons observé que le préfixe verbal explétif a une fréquence d'emploi plus dominante que celle de ha- référentiel, plus particulièrement lorsqu'il intervient comme sujet. Une raison semble justifier cet état de choses : le sujet ha explétif est un élément caractéristique d'un certain nombre d'énoncés spécifiques en usage en kirundi. Telles sont notamment les constructions présentatives ( NSHEMEZIMANA, 2016) et les constructions d'inversion explétive (NDAYIRAGIJE, 1999). Une construction d'inversion explétive est une structure dont le sujet lexical est porté en position postverbale sans qu'il soit remplacé par un autre constituant syntaxique. Or, pour une langue comme le kirundi où les fonctions syntaxiques sont obligatoirement marquées, l'emploi du préfixe explétif ha- sur le verbe se présente comme une stratégie de doter le verbe d'une marque de sujet pour combler ce vide occasionné par le déplacement du sujet du verbe dans une position non canonique. Un exemple illustrant un tel type de construction est celui en (17) ci-dessous. Ici, Le préfixe ha- intervient comme un sujet impersonnel n'ayant aucun lien avec un quelconque réfèrent locatif.

(17) Havuga uwo bubezwe.

$\begin{array}{lll}{[\text { [ha] }]^{\text {EXP- } \varnothing-v u ́ g-a ~}} & \text { u-u-ó } & \text { bu-bég-u-ye } \\ \text { SUJ } & \\ \text { EXP-PRS-parler-VF } & \text { AUG1-PP1-PRCS } & \text { SUJ14-prendre.peu-PASS-PRF.REL }\end{array}$

"Il crie généralement plus fort celui qui n’a pas essuyé une grande perte." (Umurundi, Poésie, 1960s)

Comme déjà précisé, les présentatives sont une autre catégorie d'énoncé où intervient le sujet explétif ha-, plus particulièrement celle à vocation existentielle (NSHEMEZIMANA, 2016) correspondant à celle illustrée dans (18) ci-dessous.

(18) Hari abakenyezi n'abigeme bagwaanye muri iyi mirwi yagwana.

\begin{tabular}{|c|c|c|c|c|c|c|}
\hline $\mathbf{a}]^{\mid}+a^{-}$ & -ri & & ezi & '’ & a-ba-iígeme & ba-á-gwaan-ye ${ }^{H}$ \\
\hline & & & -PN2-femme & & $\mathrm{AUG}_{2}-\mathrm{PN}_{2}$-fille & -PE-lutter-PRF.REL \\
\hline & $\mid-I-I$ & & mI-rWI & & -awaаn-a & \\
\hline $\mathrm{C}_{18}$ & AUG4-PF & EM & PN4-groupe & & J4-PE-se.battre & F.REL \\
\hline
\end{tabular}

"Il y a des femmes et des filles qui ont lutté parmi ces groupes qui se battaient." (CU101004Abaru, Paix, 2010s) 
Dans l'exemple ci-dessus, le sujet explétif ha- se trouve régulièrement associé avec le verbe -ri "être" pour constituer la forme verbale hari "il y a" introduisant ce type d'énoncé donné. Dans cet environnement syntaxique, cette forme verbale a perdu son sémantisme inhérent et fonctionne comme du support matériel pour l'expression de la construction en question. Autrement dit, il est réduit à un simple marqueur présentatif. II peut apparaître également sous la forme harihó dont le suffixe post-final -hó y confère plutôt le sens de "il existe", comme dans l'exemple en (19) ci-après :

(19) Hariho umusore yitoye aja kuresha umukobwa

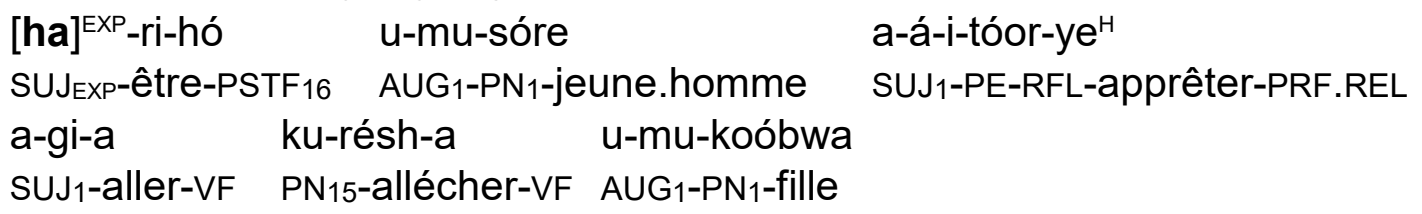

"Il y a un jeune homme qui s'est rendu à la recherche d'une fiancée."

(Kw'lsoko201140, Religion, 2010s)

Le suffixe postfinal -hó, dans (19), ne renvoie pas à un référent particulier, contrairement au suffixe locatif -hó référentiel à la classe locative 16, décrit précédemment. II n'a véritablement pas de valeur locative, bien qu'il y soit associé diachroniquement. Son emploi dans le verbe lui attribue plutôt une valeur existentielle, d'où l'appellation des présentatives en question. L'ensemble donne lieu à la forme verbale lexicalisée -rihó qui a le sens de "exister" en français. Nous pouvons aussi parler d'élément grammatical désemantisé ; ce qui lui confère, du moins dans le contexte en présence, le statut de "clitique explétif". Le corpus montre que ce suffixe est plus attesté dans ce type de marqueur présentatif. Il y est identifié à plus de $90 \%$ de ses occurrences globales.

Notons de manière générale que pour les verbes où le sujet explétif ha- est en usage simultané avec le suffixe explétif-hó, ce dernier confère au verbe donné une valeur existentielle. Ceci est aussi le cas dans l'exemple en (20) ci-après.

(20) Nooné reeró hagumehó ukweémera.

Nooné reeró [ha] ${ }^{\mathrm{EXP}}$-gum-e-hó u-ku-eémera

Ainsi donc SUJEXP-Continuer-SBJ-PSTFEXP AUG15-PN15-foi

"Ainsi donc, que la foi reste."

(UbwuzureBushasha, Religion, 1960s)

\section{b) Ha, marqueur de l'objet explétif}


Le corpus a montré que le préfixe explétif ha-peut aussi fonctionner comme objet explétif mais avec une fréquence d'emploi relativement faible. Nous en avons uniquement trouvé 33 cas sur 689 attestation (soit 3,63\%) à l'oral contre 25 cas sur 875 attestions (soit 3,77\%) à l'écrit. En outre, en emploi d'objet explétif, ha- ne peut alterner qu'avec l'unique suffixe explétif -hó, contrairement à ce que nous avons observé en (16) en rapport avec la possibilité d'alternance entre l'objet locatif et le suffixe locatif co-référent. En voici une illustration dans les exemples ci-après :

(21) Iyo umuziro wari ubafatiriye uhavuye (...)

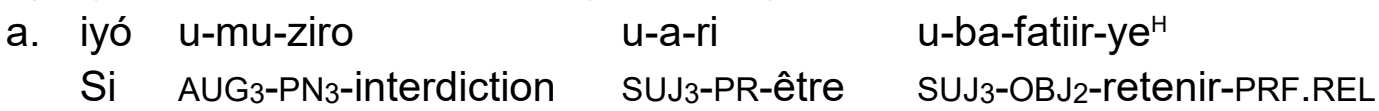

$\mathrm{u}-[\mathrm{ha}]^{\mathrm{EXP}}-\mathrm{vu}-\mathrm{ye} \mathrm{H}^{\mathrm{H}}$

SUJ3-OBJEXP-quitter-PRF.REL

"Si l'interdiction qui les retenait est levé...."

(AmategekoAgenga, Lois, 1990s)

b. Iyó u-mu-ziro

u-a-ri

u-ba-fatiir-ye ${ }^{H}$

Si AUG3-PN3-interdiction

SUJ3-PR-être

SUJ3-OBJ2-retinir-PRF.REL

u-vu-ye-hó (...)

SUJ3-quitter-PRF.REL-PSTFEXP

(22) Mu mategeko yahahora, uwupfunzwe icamaso ntiyasubira kubonana n'uwundi muntu.

a. mu ma-tégeko a-a-[ha] ${ }^{\mathrm{EXP}}-\mathrm{hór}_{-} \mathrm{a}^{\mathrm{H}}$

$L_{18}$ PN6-loi SUJ6-PR-OBJEXP-exister-VF.REL

u-wu-pfung-u-ye ${ }^{\mathrm{H}}$ icáamáaso nti-a-a-súbiir-a

AUG1-SUJ1-emprisonner-PASS-PRF.REL garde.à.vue NEG-SUJ1-PR-encore-VF

ku-bónan-a n' u-u-ndi mu-ntu

$\mathrm{PN} 15$-se.voir-VF avec $\mathrm{AUG}_{1}$-PP1-autre $\mathrm{PN}_{1}$-personne

"Parmi les lois qu'il y avait quelqu'un mis en garde à vue n'avait plus le droit d'être en contact avec quelqu'un d'autre."

(Igitabo c'amatégeko, Lois, 2000s)

b. mu ma-tégeko a-a-hór-a ${ }^{\mathrm{H}}$-[hó] ${ }^{\mathrm{EXP}} \quad(\ldots)$

LOC18 PN6-lois SUJ6-PR-OBJEXP-exister-VF.REL (...)

\section{Conclusion}

En kirundi, il existe deux types de préfixes verbaux "ha-/-ha-" en usage dans la langue. Le premier est référentiel, co-référant à la classe locative 16, et le second non référentiel, utilisé comme élément explétif dans le verbe donné. 
Sur le plan fonctionnel, ces types de préfixes ont en commun le fait qu'ils peuvent tous fonctionner comme sujet ou objet du verbe tout en gardant chacun sa propriété distinctive de référentialité ou de non référentialité. Mais, le préfixe locatif (référentiel) a la particularité de pouvoir jouer le rôle d'élément d'accord entre le verbe et son sujet locatif, lorsque ce dernier est interne à la relation syntaxique formée par le verbe et le reste de l'énoncé. En outre, la fonction morphosyntaxique de ces morphèmes détermine leur position syntaxique dans le verbe. En effet, le préfixe ha en fonction du sujet ou d'accord verbal est régulièrement en position préverbale et celui en fonction d'objet est en position préradicale.

Par rapport, à la fréquence d'emploi de ces éléments morphologiques, l'étude a montré que l'explétif ha a une dominance importante dans ses emplois surtout lorsqu'il intervient comme sujet explétif dans le verbe. Sa présence dans le verbe a des fins variées au niveau morphosyntaxique. II participe notamment au marquage des structures spécifiques en y conférant en même temps une lecture syntaxicosémantique particulière. L'exemple typique est celui des phrases présentatives existentielles dont le caractère existentiel prend origine dans le préfixe en question. Ailleurs, comme dans les structures d'inversion explétive, l'explétif ha- se trouve comme une stratégie d'attribuer le sujet au verbe l'ayant perdu à l'issue de l'opération d'inversion du sujet, laquelle opération contraint ce dernier à être délocalisé de sa position canonique, perdant ainsi sa capacité de contrôler l'accord du verbe.

Cette étude ouvre des horizons à de nouvelles perspectives au sujet des préfixes verbaux ha-/-ha-. A titre d'exemple, une étude visant les fonctions sémantiques ou discursives de ces morphèmes en kirundi constitue une bonne perspective envisageable dans les recherches futures sur cette langue bantoue.

\section{Abréviations}

$\begin{array}{llll}{[\ldots]^{H}} & : \text { ton haut marqueur de mode } & \text { PLOC } & \text { : préfixe locatif } \\ \text { ACC } & : \text { accord } & \text { PN } & : \text { préfixe nominal } \\ \text { APPL } & : \text { applicatif } & \text { POSS } & : \text { possessif } \\ \text { AUG } & : \text { augment } & \text { POT } & : \text { potentiel } \\ \text { CJ } & : \text { conjoint } & \text { PP } & : \text { préfixe pronominal } \\ \text { DEM } & : \text { démonstratif } & \text { PR } & : \text { passé récent }\end{array}$




\begin{tabular}{|c|c|c|c|}
\hline DJ & : disjoint & PRCS & : précessif \\
\hline EXP & : explétif & PRF & : perfectif \\
\hline LOC & : locatif & PRO & : pronominal \\
\hline LOC_EXT & : locatif externe & PRS & : présent \\
\hline LOC_INT & : locatif interne & PSTF & : postfinal \\
\hline NEG & : négatif & PV_EXP & : préfixe verbal explétif \\
\hline OBJ & : objet & REL & : relatif \\
\hline OBJ_EXP & : objet explétif & RFL & : réfléchi \\
\hline OBJ_PRO & : objet pronominal & SBJ & : subjonctif \\
\hline P_PréRAD & : préfixe préradical & SG & : singulier \\
\hline P_PréVRB & : préfixe préverbal & SUF_LOC & : suffixe locatif \\
\hline PA & : préfixe adjectival & SUJ & : sujet \\
\hline PASS & : passif & SUJ_EXP & : sujet explétif \\
\hline PE & : passé éloigné & SUJ_PRO & : sujet pronominal \\
\hline PERS & : persistif & VF & : voyelle finale \\
\hline PL & : pluriel & VP_LOC & : préfixe verbal locatif \\
\hline
\end{tabular}

\section{Références}

BOSTOEN, K.; MBERAMIHIGO, F.; DE SCHRYVER, G.-M. Grammaticalization and subjectification in the semantic domain of possibility in Kirundi (Bantu, JD62). Africana Linguistica, 18, 5-40, 2012.

BRESNAN, J.; MCHOMBO, S. A. Topic, pronoun, and agreement in Chichewa. Language, 63(4), 741-782, 1987.

BUKURU, D. Phrase structure and fonctional categories in kirundi sentence. University of Dar-es-salaam, 2003.

DALGISH, G. M. Locative NP's, locative suffixes, and grammatical relations. Annual Meeting of the Berkeley Linguistics Society, 2, 139-148, 1976.

DEVOS, M.; MISAGO, M.-J.; BOSTOEN, K. A corpus-based description of locative and non-locative reference in Kirundi locative enclitics. Africana Linguistica, 23, 47-83, 2017.

GIVON, T. Topic, Pronoun, and Grammatical Agreement. In Subject and Topic (Academic Press, p. 149-188.), 1976.

GUTHRIE, M. The Status of Radical Extensions in Bantu Languages. Journal of African Languages, 1(3), 202-220, 1962. 
HYMAN, L. M.; DURANTI, A. On the Object Relation in Bantu. In P. Hopper \& S. Thompson (Éds.), Synlax and Semantics : Studies in Transitivity (Vol. 15, p. 217-239). Academie Press, 1982.

LAFKIOUI, M.; NSHEMEZIMANA; E.; BOSTOEN, K. Cleft constructions and focus in Kirundi. Africana Linguistica, 22, 71-106, 2016.

MARTEN, L.; VAN DER WAL, J. A typology of Bantu subject inversion. Linguistic Variation, 14(2), 318-368, 2014.

MBERAMIHIGO, F. L'expression de la modalité en kirundi. Exploitation d'un corpus électronique. Université libre de Bruxelles, 2014.

MBERAMIHIGO, F.; DE SCHRYVER, G.-M.; BOSTOEN, K. Entre verbe et adverbe : Grammaticalisation et dégrammaticalisation du marqueur épistémique umeengo/umeenga en kirundi (bantou, JD62). Journal of African Languages and Linguistics, 37(2), 247-286, 2016.

MBERAMIHIGO, F.; DE SCHRYVER, G.-M.; BOSTOEN, K. Entre modalité et conditionnalité : L'analyse de corpus du préfixe verbal oo- en kirundi (bantou, JD62). Studies in African Linguistics, 49(2), 2020.

MAHO, J. F. NUGL Online: The Online Version of the New Updated Guthrie List, a Referential Classification of the Bantu Languages, 2009.

MEEUSSEN, A. E. Essai de Grammaire Rundi. Annales du Musée Royal du Congo Belge, 1959.

MISAGO, M.-J. Les verbes de mouvement et l'expression du lieu en kirundi (bantou, JD62) : Une étude linguistique basée sur un corpus. Université de Gand, 2018.

MISAGO, M.-J.; NSHIMIRIMANA, E.; TUYUBAHE, P. Adverbes locatifs de classe 17 ku en kirundi (JD62). Odyssey Magazine, 4, n. 2, pp 34-52, 23 août. 2019.(2), 34-52, 2019.

MORIMOTO, Y. Discourse Configurationality in Bantu Morphosyntax. Stanford University, 2000.

NDAYIRAGIJE, J. Checking Economy. Linguistic Inquiry, 30(3), 399-444, 1999.

NSHEMEZIMANA, E. Marquage morphosyntaxique des topiques en kirundi (JD62). Journal of University of Burundi, 18(1), 58-67, 2021.

NSHEMEZIMANA, E. Morphosyntaxe et structure informationnelle en kirundi : Focus et stratégies de focalisation. Université de Gand, 2016.

NSHEMEZIMANA, E.; BOSTOEN, K. The conjoint/disjoint alternation in Kirundi (JD62): A case for its abolition. In The Conjoint/Disjoint Alternation in Bantu (van der Wal, Jenneke\&Hyman, Larry M., p. 390-425). Walter de Gruyter, 2017. 
NTAHOKAJA, J.-B. Grammaire structurale du kirundi. ACCT, 1994.

SABIMANA, F. The Relational Structure of the Kirundi Verb. Indiana University, 1986.

TOGNINI-BONELLI, E. Corpus Linguistics at Work (John Benjamins.), 2001.

TUYUBAHE, P. Valence des verbes et interdependances entre lexique et syntaxe en kirundi [Thèse de doctorat]. Université de Liège, 2017.

VAN DER WAL, J. Word order and information structure in Makhuwa-Enahara. Leiden University, 2009.

VITALE, A. J. Swahili Syntax, 1981.

WALD, B. Grammar and Pragmatics in the Swahili Auxiliary Focus System. The Annual Proceedings of the Berkeley Linguistics Society, 32, 128-139, 1997.

ZERBIAN, S. Inversion structures in Northern Sotho. Southern African Linguistics and Applied Language Studies, 24(3), 2006. 